\title{
Germanica
}

\section{La jeunesse chez trois jeunes auteurs des années 90 : Th. Brussig, B. von Stuckrad-Barre et B. Lebert}

Die Jugend bei drei deutschen jungen Autoren: Th. Brussig, B. von Stuckrad-

Barre et B. Lebert

Frédéric Weinmann

\section{(2) OpenEdition}

\section{Journals}

Édition électronique

URL : http://journals.openedition.org/germanica/2160

DOI : 10.4000/germanica. 2160

ISSN : 2107-0784

Éditeur

Université de Lille

Édition imprimée

Date de publication : 30 juin 2002

Pagination : 117-127

ISBN : 9782913857070

ISSN : 0984-2632

Référence électronique

Frédéric Weinmann, «La jeunesse chez trois jeunes auteurs des années 90 : Th. Brussig, B. von Stuckrad-Barre et B. Lebert », Germanica [En ligne], 30 | 2002, mis en ligne le 16 juillet 2013, consulté le 06 octobre 2020. URL : http://journals.openedition.org/germanica/2160 ; DOI : https://doi.org/ 10.4000/germanica.2160

Ce document a été généré automatiquement le 6 octobre 2020.

(c) Tous droits réservés 


\title{
La jeunesse chez trois jeunes auteurs des années 90 : Th. Brussig, B. von Stuckrad-Barre et B. Lebert
}

\author{
Die Jugend bei drei deutschen jungen Autoren: Th. Brussig, B. von Stuckrad-
}

Barre et B. Lebert

Frédéric Weinmann

Ja, junger Autor, das ist ein hohles Attribut. Jung
und was? Gefährlich ? Unverbraucht? Naiv?
Wild? Unschuldig? Stimmt alles nicht. Wenn
Ihnen was dazu einfällt, schreiben Sie über mein
Alter, klar, warum nicht. Aber nächstes Jahr
müssen sie sich dann spätestens was anderes
einfallen lassen.
Benjamin v. Stuckrad-Barre, Livealbum, p. 94.

Dans le cadre du salon du livre de Francfort au lendemain de la nomination de Günter Grass pour le prix Nobel de littérature, le Spiegel consacrait la couverture de son numéro du 11 octobre 1999 à six «nouveaux poètes allemands », présentés comme les "petits-enfants» de l'illustre écrivain et retenus parmi beaucoup d'autres comme représentants d'une jeune génération: il s'agissait de Thomas Brussig, Karen Duve, Jenny Erpenbeck, Benjamin Lebert, Thomas Lehr et Elke Naters. L'hebdomadaire hambourgeois réfléchissait ainsi - et en même temps participait - au lancement d'un phénomène à la fois littéraire et commercial qui devint rapidement un cliché et trouva son point d'orgue lors du salon de livre de Paris en mars 2001, à savoir la diffusion de jeunes auteurs, l'affirmation d'un nouveau courant et le prétendu retour de l'Allemagne au premier plan de l'édition internationale. Il n'est pas question ici d'analyser les mécanismes de cette brillante campagne de marketing, mais en partant du constat qu'à la fin des années 1990, de jeunes écrivains de langue allemande sont parvenus à intéresser un large public, majoritairement jeune également, nous 
chercherons à déterminer l'importance que prend dans leurs ouvrages le motif de la jeunesse.

2 S'il est exclu de peser les mérites littéraires des différents auteurs pour en retenir certains plutôt que d'autres, le nombre de titres publiés par cette nouvelle génération est toutefois trop grand pour qu'on puisse les traiter tous. Face à la nécessité de faire un choix, on aurait pu par exemple porter toute son attention sur Zoë Jenny, Suissesse née en 1974 dont le premier livre, Das Blütenstaubzimmer - qui s'est vendu à 100000 exemplaires en allemand et a été traduit en français par Nicole Roche sous le titre $L a$ chambre des pollens -, décrit le désarroi des enfants nés au lendemain de 1968 et laissés à eux-mêmes par des parents anti-autoritaires et égocentriques. On aurait pu aussi s'arrêter au premier roman de Jenny Erpenbeck, Geschichte vom alten Kind - traduit cette fois par Bernard Kreiss sous le titre Histoire de la vieille enfant - dans lequel l'auteur met en scène la vie d'une orpheline de quatorze ans dans un foyer et où le motif de la jeunesse et de l'enfant sauvage sert ostensiblement de parabole du renouveau.

Mais conscient de traiter d'un phénomène de mode, nous avons décidé de retenir les trois auteurs qui nous paraissent les plus médiatisés et dont les ouvrages ont atteint des tirages exceptionnels. Il s'agit tout d'abord de Thomas Brussig, né en 1965 à Berlin Est, dont le roman Helden wie wir - paru en 1995 et traduit par Olivier Mannoni sous le titre Le complexe de Klaus - s'est vendu à plus de 200000 exemplaires en langue originale et a donné lieu à un film sorti en novembre 1999. Nous lui associons les deux benjamins de la maison Kiepenheuer \& Witsch, qui ont également fait mouche puisque les titres Soloalbum et Livealbum de Stuckrad-Barre ont atteint un tirage de 150000 exemplaires chacun tandis que le roman à caractère autobiographique de Lebert, Crazy, a dépassé à lui seul les 300000 et a été porté à l'écran, où il n'a pas eu moins de succès ${ }^{1}$.

4 Dernier avatar du mythe de l'enfant prodige, ces deux derniers incarnent peut-être plus encore que Brussig et les autres auteurs l'étrange et brève rencontre des critiques et du grand public dans un commun enthousiasme, ce point d'intersection entre la littérature et le divertissement qui semble une négation des catégories traditionnelles. Leur auto-complaisance et leur impact médiatique - depuis le film dans lequel Lebert joue son propre rôle jusqu'à Blackbox où Stuckrad-Barre met en scène la liaison que la presse à scandale lui a prêtée avec la comédienne Anke Engelke - portent à son comble la question du rapport entre l'art et le commerce et ne représentent assurément qu'un aspect de la vie culturelle de la dernière décennie, ce que d'aucuns ont appelé la Popliteratur.

5 D'autres écrivains à peine moins jeunes et à peine moins connus, mais beaucoup plus discrets et peut-être plus talentueux - tels Ingo Schulze dont les œuvres sont traduites en français ou Dirk Kurbjuweit qui vient de publier chez Nagel \& Kimche un beau roman retraçant l'amitié indéfectible qui lie deux adolescents - montrent sous un autre jour la société actuelle et leur propre génération. Mais toute éphémère, artificielle et partielle qu'elle soit, phénomène de société et phénomène de foire à la fois, la forme d'écriture qui nous paraît commune aux meilleurs best-sellers des années 1990 mérite d'être retenue comme symptomatique d'une certaine jeunesse de cette époque, qui s'y reconnait, comme image sans doute moins réaliste que fantasmée des adolescents et post-adolescents de la jeune République berlinoise.

6 Bien qu'un monde sépare un enfant de la R.D.A., un jeune homme des grandes villes de la R.F.A. et un adolescent handicapé du Sud de l'Allemagne, il se dégage en effet de Helden wie wir, Soloalbum, Livealbum et Crazy une parenté frappante. Autobiographiques 
dans leur forme, c'est-à-dire rédigés à la première personne, et directement plantés dans la réalité contemporaine grâce à des allusions permanentes aux événements historiques, à l'évocation de personnes réelles et la description d'habitudes de vie actuelles, ces récits retracent sur le mode de la dérision des épisodes et des expériences qui font apparaître la jeunesse comme une époque difficile, remplie de gênes et de doutes, mais dont la peinture tend en fait à décrire des situations scabreuses, à renverser ainsi toutes les conventions et donc à s'affirmer dans la provocation et l'insouciance.

7 Le roman de Thomas Brussig, pour présenter les ouvrages dans l'ordre chronologique, décrit le parcours d'un jeune Allemand de l'Est depuis sa naissance le 20 août 1968 jusqu'à la chute du mur - dont il s'imagine être le principal responsable. Le traducteur, Olivier Mannoni, résume l'essentiel en ces termes :

Le Complexe de Klaus est un roman picaresque et comique, où l'auteur ne recule devant aucune énormité pour écraser sous la dérision le système qui a enfermé son enfance dans un cadre terne et sage. Il y raconte l'histoire d'un gamin, qui porte le nom impossible de Klaus Uhlztscht, et découvre avec une infinie naïveté l'univers réel derrière le monde artificiel que lui ont créé ses parents : un père officier de la Stasi, une mère hygiéniste. Celle-ci traque le microbe, celui-là, le dissident ${ }^{2}$.

Dans une prétendue interview donnée à un journaliste américain, le protagoniste y retrace en effet pas à pas les souffrances de son enfance et les étapes de sa lente maturation : les premières années passées à la maison avec sa mère, les humiliations de l'école, les surprises de la colonie de vacances, l'entrée dans les services de la police politique après le baccalauréat, sa première relation avec une jeune fille, son début de carrière à la Stasi et finalement les malheurs du jeune homme dans un Berlin en pleine ébullition.

Durant tout l'ouvrage se superposent deux voix, celle du narrateur (adulte) qui rappelle ou juge les faits avec lucidité et distance critique et en même temps celle du personnage (enfant et adolescent) qui n'a pas encore les moyens de comprendre rationnellement et interprète la réalité avec la sensibilité propre à son âge. Helden wie wir n'est donc pas seulement le récit d'une jeunesse malheureuse dans le cadre de la R.D.A., mais c'est aussi et surtout le compte rendu d'une vision différente du monde que le narrateur définit lui-même dans le chapitre d'introduction, en refusant de se prendre au sérieux, mais en l'étant quand même :

Kindheit ist die Zeit ohne Zweifel. Igitt, mir ist ein Aforismus rausgerutscht! Ich verabscheue Aforismen! Besonders die eigenen. Aber auch sonst: Sie haben das gewisse Etwas für fettarschige, behäbige Zuhörer, aha, aha, interessant, so habe ich es noch gar nicht gesehen... Seit meinen Leserbriefen habe ich Übung in Aforismen, sie unterlaufen mir ständig, sie fließen aus mir raus wie Dünnpfiff. Wo war ich? Kindheit, Zweifel, ja, ein Kind muß glauben, wo es nicht versteht, aber wenn es zweifelt, hört es auf, Kind zu sein. Aforistisch, nicht wahr? Hätte garantiert die Qualifikation zum Abdruck in der Abschlußdiskussion « $\mathrm{Zu}$ jung zum Erwachsensein? Zu alt für ein Kind ? " gereicht. Die Jugend ist die Zeit der Zweifel, und wo Zweifel enden, endet Jugend. Wenn das nicht Dünnpfiff-Denken ist, ohne Konsistenz, ohne Schwere - aber mein Kopf ist voll von diesem aforistischen SchubiDubi ${ }^{3}$.

On voit d'emblée ce qui caractérise l'écriture de Brussig: le refus des grandes déclarations, le besoin d'en faire quand même et la résolution de cette contradiction par l'autodérision et l'auto-négation. C'est ainsi qu'il parvient à reculons à donner une définition claire de la jeunesse - l'époque de la vie comprise entre la foi naïve de 
l'enfant et les convictions hypocrites de l'adulte - dans un style en même temps propre à sa génération.

En partant de prémisses toutes différentes, Stuckrad-Barre en arrive à des conclusions et un résultat comparables. Ainsi, dans son premier ouvrage, il publie le carnet de bord d'un garçon d'une bonne vingtaine d'années abandonné par son amie après une liaison de quatre ans. Le narrateur est presque déjà sorti de la jeunesse : il ne vit plus chez ses parents auxquels il ne fait allusion que pour se souvenir qu'ils se plaignent de ne pas le voir assez souvent, il n'est plus étudiant et il a un travail. Pourtant, même s'il a conscience d'être plus vieux que les garçons de quatorze ans qu'il voit dans un magasin (les jeunes au sens propre), il se démarque aussi de ceux qui ont des enfants (de vrais adultes) et sait bien qu'il en est encore à l'âge où l'on joue au grand, comme il le dit de son ami Alf :

Die Gastgeber sind zehn Jahre älter als wir und haben gerade eine frische Wohnung bezogen. [...] Das Paar (wissen auch nichts zu sagen) ist vollkommen übertrieben glücklich, und - völlig klar! - ein Kind haben sie auch, und das darf sogar noch wach bleiben, weil es nachher ja Pizza gibt für alle, und da wollen wir heute mal eine Ausnahme machen. Wir geben unseren Wein ab und sagen, daß wir später wiederkommen, wichtiger Termin noch und die ganze Scheiße, und zack sitzen wir wieder bei Alf. Er mixt uns einen Martini-Cocktail und spielt erwachsen ${ }^{4}$.

Il est donc difficile de savoir exactement ce qu'il entend par jeunesse car le concept ne se laisse pas réduire à une simple formule, mais qu'il se subdivise constamment en préados, adolescents, jeunes adultes et vieux. Cet éclatement atteint son comble lors d'une parodie de talk-show consacrée au sujet «Culture jeune et médias » dans laquelle les avis les plus divers se perdent au milieu de situations absurdes ${ }^{5}$. Bien loin de donner une réponse catégorique ou de s'élancer dans une longue période pour défendre ou attaquer la génération dont il fait encore partie tout en l'observant déjà avec distance, l'écrivain met ici en scène la façon de penser des gens de son âge.

Au début ce passage, pendant le trajet en train, l'influence des médias sur la jeunesse est incarnée par un jeune handicapé enfermé dans l'univers sonore de son walkman, la fin des idéologies et la montée de l'individualisme typiques de cette génération est mentionnée au milieu d'autres clichés tirés de l'actualité et le rejet du monde adulte se traduit par la fausse surprise avec laquelle le narrateur prétend découvrir qu'existent encore ces discussions vieilles comme le monde. Puis un groupe d'adolescents complètement saouls qui se remémorent bruyamment les moments épiques de la nuit précédente lui rappelle ses propres voyages scolaires et lui fait penser que la jeunesse, c'est-à-dire visiblement l'adolescence, n'a pas changé

Dans les huit pages suivantes, il continue sans en avoir l'air de traiter le sujet qu'il s'est proposé, disséminant quelques affirmations au sujet de la jeunesse dans le tableau retraçant son arrivée sur les lieux et la discussion animée qui se déroule sur le podium. Après avoir présenté les différents participants, il décrit l'abominable chaos d'un débat où s'affrontent sans compromis plusieurs générations ${ }^{7}$. Au bout du compte, la description réaliste et par là même comique des stériles talk-shows contemporains dans lesquels l'effet produit, le spectacle, compte plus que le contenu de la discussion permet indirectement à l'auteur d'exposer sa conception de la jeunesse, c'est-à-dire de défendre la génération de la Love parade qui provoque celle du Rock \& Roll et s'affirme par le biais d'un langage pour le moins familier. le plus clairement ce qui caractérise l'image de la jeunesse dans Soloalbum : derrière le 
même goût de la dérision que celui qu'on notait chez Klaus Uhltzscht, c'est-à-dire la tendance à énoncer des convictions et des idées importantes par le biais de l'anecdote et de la plaisanterie, la jeunesse apparaît comme le moment où l'individu commence à se poser des questions sur le sens de l'existence, ou plus exactement celui où il se pose vraiment ces questions sans se satisfaire des réponses convenues qui appartiennent au monde des adultes :

In der Pubertät überkommt einen zum ersten Mal der Gedanke, daß das ständige An- und Ausziehen, Gewinnen und Verlieren, Schmutzigmachen-Saubermachen, Ver- und Entlieben, Kaufen und Verkaufen - daß es einen zum Wahnsinn treibt. Man fragt dann pathetisch: WOZU ? Und ist damit auch Teil der Soße, das ist ja vorgesehen, daß man sich so benimmt, eine Zeitlang. Und der Gedanke allein ist Pubertät, wird von der Umgebung nachsichtig abgewiegelt, irgendwann auch von dir selbst. Schlimm, daß er trotzdem bleibt 8 .

Dans l'ouvrage suivant, où il dépeint en douze chapitres les différentes étapes d'une tournée de conférences qu'un jeune auteur entreprend à travers l'Allemagne et où il médite avant tout sur le rôle de la littérature et de l'écrivain à la fin $\mathrm{du} \mathrm{xx}^{\mathrm{e}}$ siècle, Stuckrad-Barre évoque aussi à plusieurs reprises le thème de la jeunesse. Son narrateur est à nouveau un jeune homme entre deux âges, à la frontière entre les adolescents qui le voussoient et les adultes qui le voussoient également.

S'il évoque dès les premières lignes de l'ouvrage son statut intermédiaire entre quarante enfants de neuf ans et neuf parents de quarante ans parce qu'il commence sa tournée dans une bibliothèque où l'on met à sa disposition «ein GANZ altes Mikrophon » qu'il imagine avoir déjà servi à Ernst Jünger pendant la Première Guerre mondiale et une chaîne Hi-fi comme celle que les adolescents recevaient en cadeau pour leur communion dans les années 1980 , c'est à l'occasion de son huitième "Show " qu'il aborde le plus précisément les problèmes de générations et offre une belle description des signes extérieurs de jeunesse.

Il raconte en effet que deux lycéennes l'invitent à partager leur soirée et l'emmènent tout d'abord chez l'une d'entre elles. C'est pour le narrateur l'occasion de découvrir la chambre de la jeune fille, qui porte à la fois les traces de l'enfant qu'elle n'est plus, les empreintes de la puberté et les signes avant-coureurs de l'adulte de demain. Par le biais de cette description, la jeunesse est présentée comme une époque de métamorphose où l'individu n'est pas encore autonome, la personnalité pas encore achevée et où tout se détermine encore par rapport aux parents. Ce n'est pas seulement l'âge où l'on commence à mentir de façon conséquente, à fumer du tabac ou d'autres substances et à avoir des relations sexuelles, mais c'est surtout celui où l'on dissimule et où l'on a honte, un âge où le comportement n'est pas encore cohérent et les goûts, pas encore tout à fait assumés, c'est-à-dire l'âge où l'on cherche.

19 Enfin, si les ouvrages de Brussig et Stuckrad-Barre offrent de nombreuses réflexions sur la jeunesse, celui de Benjamin Lebert est tout entier consacré à ce sujet et regorge par conséquent de témoignages et de formules sur cette époque de la vie. Ce récit autobiographique, dont le narrateur se présente sous le nom de l'auteur, raconte l'arrivée et le séjour d'un jeune handicapé moteur dans un pensionnat où l'inscrivent ses parents après avoir essayé déjà quatre écoles. Pour le garçon, l'essentiel se passe en dehors de la salle de classe; le narrateur rapporte les expériences qu'il fait en compagnie de cinq autres écoliers et en particulier avec Janosch, son camarade de chambrée et le meneur de la bande : boire, fumer, draguer, faire les fous, faire l'amour, faire le mur. Son handicap passe ainsi au second plan quand il découvre que chacun a 
du mal à grandir ; le pensionnat devient une véritable parabole de la jeunesse, période de transition entre l'enfance et l'âge adulte, sas entre la maison et le monde extérieur.

On pourrait multiplier les citations où les adolescents réfléchissent à leur situation de manière à la fois naïve et ironique, oscillant entre l'enthousiasme et les plaintes, comme lorsque le narrateur trouve que la jeunesse est la plus belle époque de la vie ${ }^{10}$. Mais on remarquera plutôt que la longue succession de commentaires du narrateur ou de dialogues avec ses compagnons débouche non seulement sur la même idée que chez les deux précédents auteurs, mais aussi sur la même façon de l'exprimer. Cela se vérifie en particulier au moment où l'un des camarades déclare que la jeunesse est «scheiße » et qu'un autre la définit comme une recherche de sens :

« Ich glaube, wir befinden uns gerade in der Phase, wo wir noch den Faden finden müssen. Und wenn wir den Faden gefunden haben, sind wir auch anspruchsvoll. »

«Das verstehe ich nicht », bemerkt Florian entrüstet. «Was sind wir denn, bevor wir anspruchsvoll sind?»

«Vorher sind wir, so glaube ich, Fadensuchende. Die ganze Jugend ist ein einziges großes Fadensuchen. "

" Die Jugend ist trotzdem scheiße », antwortet Janosch"

21 On retrouve donc chez Lebert ce besoin de méditer sans se prendre au sérieux, cette opposition stylistique entre "Fadensuchen » et "Scheiße», ce mélange volontaire et systématique de réflexions philosophiques ou métaphysiques et d'expressions familières et humoristiques qui frappe déjà chez Brussig et Stuckrad-Barre. Tous trois définissent en fin de compte la jeunesse comme l'époque où les certitudes de l'enfant prennent fin et les convictions de l'adulte pointent à l'horizon: pour Brussig, c'est l'heure des doutes ; pour Stuckrad-Barre, le moment où l'on s'interroge sur le pourquoi de la vie; pour Lebert, une recherche de fils conducteurs. Mais non moins que le contenu de cette définition, c'est la manière dont il la formule, un mode ambigu mêlant l'ironie au sérieux, un refus du lyrisme au profit d'un volontaire prosaïsme, qui rapproche ces jeunes auteurs.

De fait, leurs récits donnent l'image d'une jeunesse qui abhorre la poésie et les grandes envolées, qui ignore les tabous et la retenue, mais qui recourt au contraire sans cesse à la provocation, à moins qu'elle ne fasse simplement profession de franchise. Cela se vérifie tout particulièrement à propos du grand sujet de l'adolescence, qu'ils abordent tous les trois sans fard: la sexualité. Aux antipodes de la pudeur convenue, les jeunes auteurs de la Popliteratur entrent non seulement dans les détails, mais recourent à une langue particulièrement familière.

On pourrait ainsi comparer la manière dont Klaus Uhltzscht raconte sa découverte de mystères de la procréation dans le dortoir de la colonie de vacances ${ }^{12}$ au récit que fait Benjamin Lebert de sa première relation sexuelle. Non seulement la colonie de vacances joue pour le petit Allemand de l'Est le même rôle que l'internat pour le jeune handicapé, mais surtout les deux écrivains se ressemblent dans leur manière d'aborder un sujet essentiel au moment de la puberté. C'est par exemple en ces termes que Lebert fait le bilan de son expérience :

Wie lauten doch gleich die ganzen Sprüche mit dem ersten Mal ? Nach dem ersten Mal wäre man ein Mann? Da stehe man auf eigenen Füßen? Vorbei sei es mit der milden Jugend? Man wäre nun erwachsen ? Hm? Mein erstes Mal ist nun vorbei. Und ich fühle mich noch immer wie ein kleiner Hosenscheißer. Das ist, glaube ich, auch ganz gut so. Ich will gar nicht erwachsen werden. Ich will ein ganz normaler Junge bleiben. Meinen Spaß haben. Mich, wenn nötig, bei meinen Eltern verstecken. Und das soll jetzt alles vorbei sein? Nur weil ich meinen Schwanz in das geile Loch 
von Marie gesteckt habe? Das hat doch sowieso niemand gesehen. Ich werde es auch niemandem erzählen ${ }^{13}$.

24 Les jeunes auteurs traitent l'une des questions principales de la jeunesse sur un mode qui leur paraît propre, c'est-à-dire de manière volontairement non littéraire, non poétique, non sentimentale, non lyrique, mais au contraire directe, familière et cocasse - de manière opposée à celle des grands romanciers du début du siècle, par exemple, qui n'abordent encore l'éveil de la sensibilité ou la confusion des sentiments qu'en termes choisis.

Moins concerné par la découverte des plaisirs de la chair en raison de son âge, le narrateur de Soloalbum évoque lui aussi la sexualité de la jeunesse sur ce ton d'évidence qui paraît propre à sa génération. Après avoir avoué sans la moindre gêne le plaisir que lui apporte la masturbation, il décrit par exemple la liberté des jeunes vivant sur l'île où il passe ses vacances et raconte comment il a assouvi ses besoins avec une jeune fille :

Wir liegen dann irgendwann morgens am Strand unter einer Schaukel und ficken ein bißchen. Ficken ist ja wirklich vollkommen schwierig, wenn man es monatelang nicht getan hat. Es geht trotzdem relativ gut. Daß ich dabei und danach nicht einsehen mag, daß dies einfach eine schöne, aber eben gerade schöne, weil einmalige Angelegenheit ist, bereitet mir etwas Sorge, aber erst anderntags, als mir auffällt, daß ich ihr meinen Ring geschenkt habe. Das mußte ja nicht sein. Aber das andere, das Ficken, das mußte sein ${ }^{14}$.

Ces quelques citations suffisent à mettre en évidence le ton propre à cette jeunesse, une génération qui écrit comme elle parle - et qui parle cru! Finalement, les actions rapportées par les écrivains sont sans doute moins significatives que le fait qu'ils les évoquent et la manière dont ils les présentent : ils ne sont sans doute pas les premiers à faire de telles expériences, mais ils sont peut-être les premiers à faire ce choix littéraire, c'est-à-dire à refuser la belle écriture au profit de l'expression directe.

Brussig s'explique sur cette option dès la première page de son ouvrage; après une douzaine de lignes en italiques qui pastichent le début d'une autobiographie traditionnelle, il prend directement la parole et énonce son incapacité à écrire de cette manière. Il prétend avoir passé en vain deux ans à rédiger un témoignage dans le style qu'il se doit et se demande combien de temps il aurait encore essayé si un journaliste américain n'était venu l'interviewer ${ }^{15}$. C'est donc une déclaration de guerre à la culture et à la grande littérature qui ouvre et justifie son premier roman, dont chaque chapitre est censé correspondre à une nouvelle cassette et dont le style est ostensiblement oral.

Il apporte ainsi l'image d'une jeunesse qui entend s'affirmer dans le rejet des valeurs établies, de la tradition et du sérieux. La même disposition d'esprit marque les ouvrages des deux autres auteurs : comme lui, ils adorent l'argot, les phrases nominales, les jeux de mots, les anglicismes, les chiffres, les mots écrits en majuscules, les italiques et ce refus du style est justement leur style ; il s'agit, en fait, d'une écriture qui s'adresse à ceux qui ne lisent pas - aux jeunes d'aujourd'hui.

Benjamin Lebert lui aussi énonce très clairement ce parti pris qu'il met non dans la bouche du personnage qui porte son nom, mais dans celle du meneur Janosch au cours d'une discussion passionnée qu'ils ont après avoir lu Le vieil homme et la mer. À la question de savoir ce qu'est la littérature, le jeune garçon explique à son camarade que c'est une écriture où l'on a le sentiment à chaque paragraphe que tout sonne juste et que l'on aurait agi ou pensé exactement comme le personnage. Benjamin lui demande de manière faussement naïve d'où il tient cette définition et pense qu'un professeur de littérature ne serait pas d'accord, ce qui conduit Janosch à proclamer son indifférence à 
l'égard de l'opinion des adultes et à exiger sans ambages le retour au simple plaisir de la lecture :

Davon verstehen nicht einmal Leute etwas, die etwas davon verstehen müßten. Warum machen wir uns also Gedanken darüber? Laß uns einfach lesen. Aus Freude am Lesen. Und aus Freude am Verstehen. Und laß uns nicht darüber nachdenken, ob es Literatur ist oder nicht. Das können andere tun. Wenn es tatsächlich Literatur ist, dann um so besser. Wenn nicht, dann ist es auch scheißega ${ }^{16}$.

La jeunesse est donc plus qu'un motif de la popliteratur, c'est son essence même puisqu'elle se nourrit d'une provocation qui tient de la crise d'adolescence. Cette façon d'écrire contre la littérature des professeurs, c'est-à-dire contre la littérature qu'on lit à l'école, la littérature des adultes est typique de «jeunes auteurs »- ce qui n'est pas un qualificatif vide de sens comme le prétend Stuckrad-Barre, puisque juvénile veut dire ici provocateur.

\section{NOTES}

1. Cf. «Bölls Enkel : Wo bleibt das nächste Buch?», dans Spiegel Online - 23. August 2000.

2. Olivier Mannoni, «Thomas Brussig », dans Kerstin Behre/Petra Metz (dir.), Jetzt-Autoren - Ils écrivent en allemand. Paris, Pauvert, 2001, p. 30-32, p. 30-31.

3. Thomas Brussig, Helden wie wir. Berlin, Volk und Welt, 1995, p. 17.

4. Benjamin von Stuckrad-Barre, Soloalbum. Köln, Kiepenheuer und Witsch, 1998, p. 73.

5. Cf. ibid., p. 140-141.

6. Cf. ibid., p. 142.

7. Cf. ibid., p. 146-147.

8. Cf.ibid., p. 46.

9. Benjamin von Stuckrad-Barre, Livealbum. Köln, Kiepenheuer \& Witsch, 1999, p. 146.

10. Cf. ibid., p. 56-57.

11. Ibid., p. 64-65.

12. Cf. Helden wie wir, op. cit., p. 62-63.

13. Crazy, op. cit., p. 81-82.

14. Soloalbum, op. cit., p. 128-129.

15. Cf. Helden wie wir, op. cit., p. 5-6.

16. Crazy, op. cit., p. 142-143.

\section{RÉSUMÉS}

Trois jeunes auteurs on été retenus, sur la base des tirages, pour représenter le courant qui est parvenu à percer à la toute fin $d u x^{e}$ siècle sous le nom de Popliteratur: Thomas Brussig, Benjamin von Stuckrad-Barre et Benjamin Lebert. Leurs premiers romans présentent de 
nombreux points communs et le rôle qu'y joue le motif la jeunesse, en particulier, est tout à fait comparable dans Le complexe de Klaus, Soloalbum, Livealbum et Crazy. Non seulement les trois écrivains en donnent une définition similaire - la jeunesse est le moment du doute, des questions sur le pourquoi de la vie, de la « recherche de fils conducteurs »-, non seulement ils rapportent des expériences identiques - par exemple la découverte de la sexualité -, mais surtout ils recourent à un style propre à la jeunesse : un style parlé, familier, volontairement non poétique, caractéristique de la provocation et de la crise d'adolescence et essentiel à ce mouvement littéraire.

Drei Jungautoren wurden aufgrund der hohen Auflage als Vertreter der am Ende des 20. Jahrhunderts unter dem Namen Popliteratur bekanntgewordenen Strömung in Betracht gezogen : Thomas Brussig, Benjamin von Stuckrad-Barre und Benjamin Lebert. Ihre Erstlinge haben viel gemeinsam und insbesondere die Rolle des Motivs Jugend ist in Helden wie wir, Soloalbum, Livealbum und Crazy durchaus vergleichbar. Alle drei Schriftsteller geben nicht nur eine ähnliche Definition - die Jugend sei die Zeit der Zweifel, der Fragen nach dem Wozu des Lebens, der «Fadensuche»-, sie erleben nicht nur identische Erfahrungen - zum Beispiel die Entdeckung der Geschlechtlichkeit -, sondern sie verwenden auch einen der Jugend eigenen Stil : einen gesprochenen, umgangsprachlichen, absichtlich unpoetischen Stil, kennzeichnend für Provokation und pubertätstypische Krise und wesentlich für diese literarische Bewegung.

\section{AUTEUR}

\section{FRÉDÉRIC WEINMANN}

Lycée Watteau de Valenciennes 